\title{
Ethnomedicinal and ecological status of plants in Garhwal Himalaya, India
}

Munesh Kumar ${ }^{1 *}$, Mehraj A Sheikh and Rainer W Bussmann²

\begin{abstract}
Background: The northern part of India harbours a great diversity of medicinal plants due to its distinct geography and ecological marginal conditions. The traditional medical systems of northern India are part of a time tested culture and honored still by people today. These traditional systems have been curing complex disease for more than 3,000 years. With rapidly growing demand for these medicinal plants, most of the plant populations have been depleted, indicating a lack of ecological knowledge among communities using the plants. Thus, an attempt was made in this study to focus on the ecological status of ethnomedicinal plants, to determine their availability in the growing sites, and to inform the communities about the sustainable exploitation of medicinal plants in the wild.

Methods: The ecological information regarding ethnomedicinal plants was collected in three different climatic regions (tropical, sub-tropical and temperate) for species composition in different forest layers. The ecological information was assessed using the quadrate sampling method. A total of 25 quadrats, $10 \times 10 \mathrm{~m}$ were laid out at random in order to sample trees and shrubs, and 40 quadrats of $1 \times 1 \mathrm{~m}$ for herbaceous plants. In each climatic region, three vegetation sites were selected for ecological information; the mean values of density, basal cover, and the importance value index from all sites of each region were used to interpret the final data. Ethnomedicinal uses were collected from informants of adjacent villages. About 10\% of inhabitants (older, experienced men and women) were interviewed about their use of medicinal plants. A consensus analysis of medicinal plant use between the different populations was conducted.
\end{abstract}

Results: Across the different climatic regions a total of 57 species of plants were reported: 14 tree species, 10 shrub species, and 33 herb species. In the tropical and sub-tropical regions, Acacia catechu was the dominant tree while Ougeinia oojeinensis in the tropical region and Terminalia belerica in the sub-tropical region were least dominant reported. In the temperate region, Quercus leucotrichophora was the dominant tree and Pyrus pashia the least dominant tree. A total of 10 shrubs were recorded in all three regions: Adhatoda vasica was common species in the tropical and sub-tropical regions however, Rhus parviflora was common species in the sub-tropical and temperate regions. Among the 33 herbs, Sida cordifolia was dominant in the tropical and sub-tropical regions, while Barleria prionitis the least dominant in tropical and Phyllanthus amarus in the sub-tropical region. In temperate region, Vernonia anthelmintica was dominant and Imperata cylindrica least dominant. The consensus survey indicated that the inhabitants have a high level of agreement regarding the usages of single plant. The index value was high (1.0) for warts, vomiting, carminative, pain, boils and antiseptic uses, and lowest index value (0.33) was found for bronchitis.

Conclusion: The medicinal plants treated various ailments. These included diarrhea, dysentery, bronchitis, menstrual disorders, gonorrhea, pulmonary affections, migraines, leprosy. The ecological studies showed that the tree density and total basal cover increased from the tropical region to sub-tropical and temperate regions. The species composition changed with climatic conditions. Among the localities used for data collection in each climatic region, many had very poor vegetation cover. The herbaceous layer decreased with increasing altitude,

\footnotetext{
* Correspondence: muneshmzu@yahoo.com

'Department of Forestry, HNB Garhwal University, Srinagar Garhwal,

Uttarakhand, India

Full list of author information is available at the end of the article
} 
which might be an indication that communities at higher elevations were harvesting more herbaceous medicinal plants, due to the lack of basic health care facilities. Therefore, special attention needs to be given to the conservation of medicinal plants in order to ensure their long-term availability to the local inhabitants. Data on the use of individual species of medicinal plants is needed to provide an in-depth assessment of the plants availability in order to design conservation strategies to protect individual species.

\section{Background}

The Indian Himalayan Region (IHR) has long been a source of medicine for the millions of people of this region as well as people living in other parts of India. At present, the pharmaceutical sector in India is making use of 280 medicinal plant species, of which 175 are found in the IHR [1].

The northern part of India harbors a great diversity of medicinal plants because of the majestic Himalayan range. So far, about 8000 species of angiosperms, 44 species of gymnosperms, and 600 species of pteridophytes have been reported in the Indian Himalaya [2]. Of these, 1748 species are used as medicinal plants [3], and the maximum number of species used as medicines has been reported from Uttarakhand [4]. Of these, sixtytwo are endemic to the Himalaya.

In India, the native people exploit a variety of herbals for effective treatment of various ailments. The plant parts used, preparation, and administration of drugs vary from place to place [5]. Indigenous knowledge is as old as human civilization, but the term ethnobotany was coined by an American botanist, John Harshburger [6], who understood the term to mean the study of the plants used by primitive and aboriginal people. Since time immemorial, plants have been employed by traditional medicine in different parts of the world. According to the World Health Organization (WHO), as many as $80 \%$ of the world's people depend on traditional medicine to meet their primary health care needs. There are considerable economic benefits stemming from the development of indigenous medicine and the use of medicinal plants for the treatment of various diseases [7]. Medicinal plants have traditionally occupied an important position in the socio-cultural, spiritual, and health arena of rural and tribal India. India has one of the oldest, richest, and most diverse systems of traditional medicine. The use of plants to cure diseases is an age-old practice. The preparation of locally available medicinal plants remains an important part of health care for humans, especially for people living in rural areas, where people lack access to modern medicine facilities, and are unable to afford synthetic drugs due to its high cost. The forests of India have been the source of invaluable medicinal plants since man became aware of the preventive and curative properties of plants and started using them for human health care.
The old Indian Systems of Medicine (ISM) are among the most ancient medical traditions known, and derive maximum formulations from plants and plant extracts found in the forests. About 400 plants are used in the regular production of Ayurvedic, Unani, Siddha, and tribal medicine. About $75 \%$ of these are taken from tropical forests and $25 \%$ from temperate forests. Thirty (30) percent of ISM preparations are derived from roots, $14 \%$ from bark, $16 \%$ from whole plants, $5 \%$ from flowers, $10 \%$ from fruits, $6 \%$ from leaves, $7 \%$ from seeds, $3 \%$ from wood, $4 \%$ from rhizomes, and $6 \%$ from stems. Fewer than $20 \%$ of the plants used are cultivated [8].

The occurrence of diverse ecosystems along altitudinal gradients form the tropical to the temperate and alpine zones with its associated impressive array of species and genetic diversity make India one of the 12 mega-biodiversity countries of the world. Forest represents one of the dominant components of the vegetation of India and forest floras constitute an invaluable reserve of economically important species, harboring traditional varieties and wild relatives of many crops. The wide range of plant species help to provide for people's needs, including the need for medicines.

The changing situation in the various ecological zones, especially the loss of habitat, habitat fragmentation, and habitat degradation is the major threat to plant diversity of the region. In those areas, where human population density is highest, most of the original habitats have already been destroyed, and many of the important medicinal plant species have been lost. The demand for housing, agriculture, and tourism development is also high. Degradation caused by an increase in human activities related to the growing population, and the lack of serious efforts to counteract them is an important concern. Human destruction of natural habitats, migration of human population, invasive species, the growing demand for natural resources and the lack of adequate training on the subject of biodiversity, all these factors are accelerating the loss of plant species. Along with the disappearance of plants from the area, traditional knowledge is also being lost.

The importance of ethnobiological knowledge for suggesting new paths in scientific research on ecology and conservation monitoring, has received much attention in resource management $[9,10]$. International agencies such as the World Wildlife Fund (WWF) and UNESCO as 
part of their people and plants initiative, have also promoted research on ethnobotanical knowledge and the integration of people's perceptions and practices in resource management at the local level [11]. Incorporation into biological and ecological studies of local-use patterns and of the social and institutional background that guides the relationships between people and nature, has led to a greater understanding of the relationship between social and ecological dynamics [12].

In the Himalayan region, which is rich in floral diversity, plants are used by the local inhabitants for their daily needs, even as they exploit the forests for different industrial purposes. The people of the Himalayan region are well aware of the traditional use of medicinal plants, but the ecological distribution of the species in the areas surrounding human habitat tell us the rate of its utilization for sustainable long-term use. Although many studies have been carried out on the ethnomedicinal uses of the plants described from the different parts of India and elsewhere [13-20]. However, there have been few ecological studies of medicinal plants in the Himalayan region in general, and none in Garhwal Himalaya. The present study was conducted to understand the ethnomedicinal and ecological status of plants in the region. The study focused on the following: 1). The use of medicinal plants by local inhabitants for various ailments. 2) The ecological status, presence and availability of medicinal plants around the villages for the villagers needs. 3) The level of exploitation by the local inhabitants and possible sustainable conservation measures.

\section{Materials and methods \\ Details of study area}

Ecological information about medicinal plant species was collected in three different climatic regions of Garhwal Himalaya: tropical, sub-tropical, and temperate regions at an average altitude of 350, 1100, and $2300 \mathrm{~m}$ a.m.s.l. (Figure 1), and their medicinal use was documented. The tropical region was primarily flat with a few south west facing hills. The sub-tropical region also faced toward south west. The temperate sites were south east facing. The summer season in the tropical region is very hot and temperatures range between 18$24^{\circ} \mathrm{C}$. In sub-tropical region, which is mildly hot in the summer season, temperatures range between $17-23^{\circ} \mathrm{C}$, and in temperate region temperatures range between 7 $15^{\circ} \mathrm{C}$, with some days below freezing in winter (October to February). The tropical region is part of the Pauri Garhwal district in the foothill region of Garhwal Himalaya. The sub-tropical and temperate regions are in Tehri Garhwal district. The total population of the villages was 1140 inhabitants in the tropical, 374 in the sub-tropical and 464 temperate regions respectively. Ten percent of the population $(114,38$ and 47) was interviewed. Further details of the regions are given in Table 1.

\section{Data collection and analysis Vegetation}

Ecological data indicating the species composition in different forest layers were collected from each region. The species composition (Table 2) was assessed with the help of quadrate sampling method. A total of 25, $10 \times 10 \mathrm{~m}$ quadrats were selected randomly to assess trees and shrubs, and 40, $1 \times 1 \mathrm{~m}$ quadrats were used for herbaceous plants. The vegetation data were quantitatively analyzed for density, total basal cover (TBC) [21], and the importance value index (IVI) was calculated as the sum of relative frequency, relative density and relative dominance [22]. In each climatic region, three sites were selected, and the mean values of density, basal cover, and importance value index from all sites of each region were used to interpret the final data.

\section{Ethnomedicinal inventory}

Information on plants with ethnomedicinal uses was collected from informants living in villages adjacent to the surrounding forest. After establishing oral prior informed consent in village meetings, about $10 \%$ of the inhabitants were interviewed about their dependence on the forest for various products, especially for medicinal purposes. The informants were randomly selected and included older men and women, well versed in the identification of plants, who regularly used and visited the forests since their childhood and used plants to cure various ailments. In the initial selection of informants younger participants were considered, but were later excluded because initial interviews indicated that they did not have much knowledge about medicinal plant use. The interviews were conducted in the local dialect to avoid translation problems. During the interviews structured questionnaires were used to obtain information on medicinal plants, including the local name of the plant, name of the disease for which a particular plant was used, part of the plant used etc. The informants were asked to show the plants in their natural habitat. Specimens of all plants were then collected and identified at the Garhwal University Herbarium (GUH), using [23].

\section{Consensus survey}

A consensus survey was conducted based on peoples opinion on the number of plants used for a particular ailment. The consensus factor (Fic) was used to test the homogeneity of the informant's knowledge according methods described by Trotter and Logan [24] and Ragupathy et al. [25] 


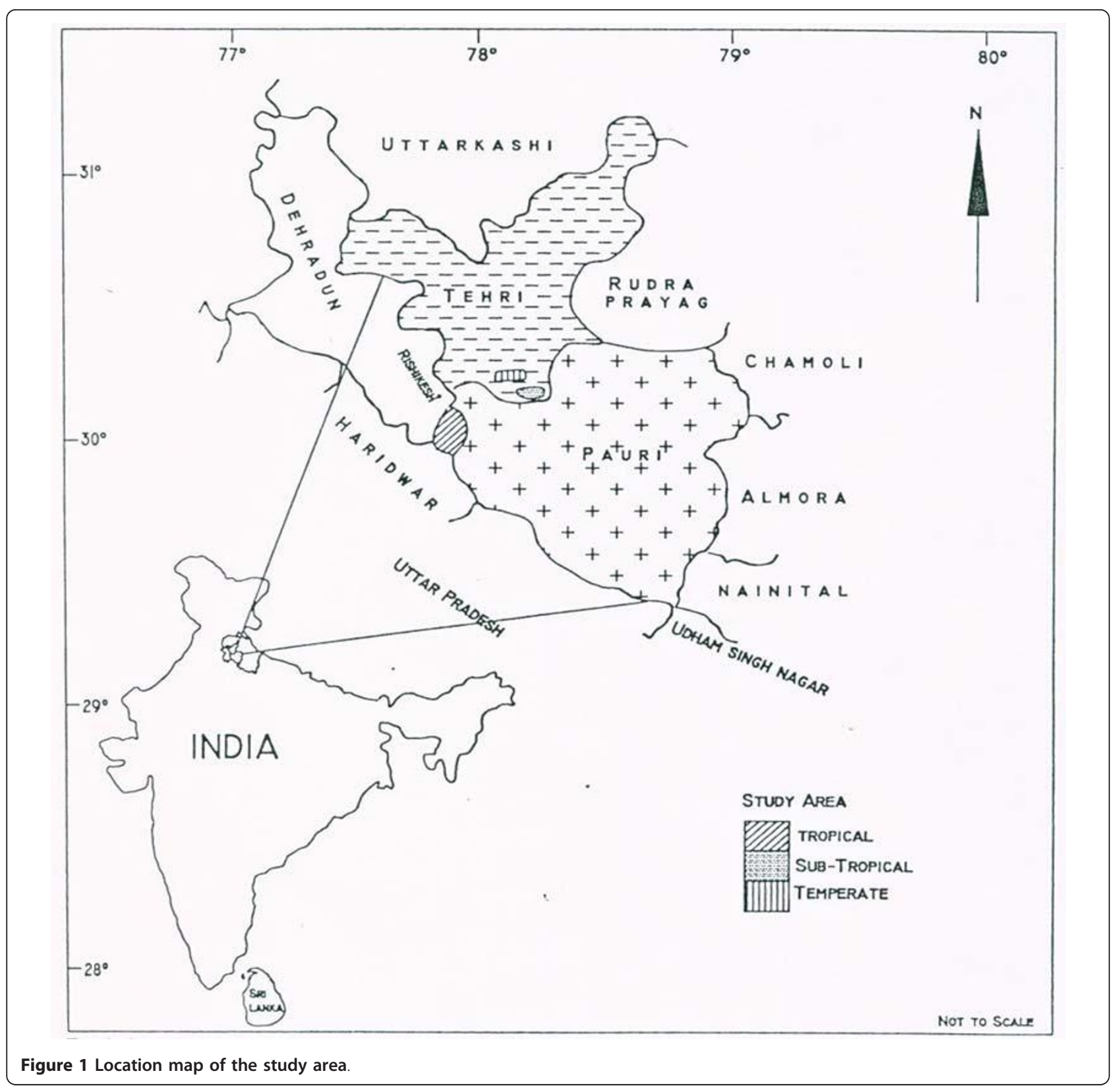

Table 1 Description of study area

\begin{tabular}{llll}
\hline Parameter & Tropical & Sub-tropical & Temperate \\
\hline Location & $30^{\circ} 6^{\prime} \mathrm{N}, 78^{\circ} 24^{\prime} \mathrm{E}$ & $30^{\circ} 29^{\prime} \mathrm{N} 78^{\circ} 24^{\prime} \mathrm{E}$ & $30^{\circ} 22^{\prime} \mathrm{N} 78^{\circ} 23^{\prime} \mathrm{E}$ \\
Altitude (m.a.s.l.) & 350 & 1100 & 2300 \\
Aspect & South West & South West & South East \\
Temperature (mean annual) & $24^{\circ}$ & $17^{\circ}-23^{\circ}$ & $7^{\circ}-15^{\circ}$ \\
Precipitation (mm) & 1350 & 960 & 1600 \\
Human population & 1140 & 374 & 464 \\
Total informants & 114 & 38 & 47 \\
Average family size & 6 & 5 & 6
\end{tabular}


Table 2 Density, TBC (total basal cover), IVI (importance value index) of ethnomedicinal plants

\begin{tabular}{|c|c|c|c|c|c|c|c|c|c|c|c|c|}
\hline \multirow{2}{*}{$\frac{\text { Species }}{\text { Trees }_{\left(\mathrm{ha}^{-1}\right)}}$} & \multirow[t]{2}{*}{ Family } & \multirow[b]{2}{*}{ Ethnomedicinal uses } & \multirow[b]{2}{*}{$\begin{array}{l}\text { Part } \\
\text { used }\end{array}$} & \multicolumn{3}{|c|}{ Tropical } & \multicolumn{3}{|c|}{ Sub-tropical } & \multicolumn{3}{|c|}{ Temperate } \\
\hline & & & & Density & TBC & IVI & Density & TBC & IVI & Density & TBC & IVI \\
\hline $\begin{array}{l}\text { Acacia catechu (L. } \\
\text { f.) Willd. }\end{array}$ & Fabaceae & $\begin{array}{l}\text { digestive purposes, respiratory } \\
\text { diseases, diarrhea, dysentery, } \\
\text { bronchitis, menstrual disorder }\end{array}$ & $W, B$ & 88 & 2.08 & 29.22 & 106 & 2.40 & 41.20 & - & - & - \\
\hline $\begin{array}{l}\text { Aegle marmelos } \\
\text { (L.) Corrêa }\end{array}$ & Rutaceae & digestive disorders & $\mathrm{F}$ & 52 & 0.74 & 19.19 & 88 & 1.34 & 34.08 & - & - & - \\
\hline Cassia fistula $\mathrm{L}$. & Fabaceae & $\begin{array}{l}\text { antiseptic, asthma, respiratory } \\
\text { disorder }\end{array}$ & $F, B$ & 56 & 1.068 & 17.39 & - & - & - & - & - & - \\
\hline $\begin{array}{l}\text { Holarrhena } \\
\text { antidysenterica (L.) } \\
\text { Wall. ex A. DC. }\end{array}$ & Apocynaceae & Dysentery, febrifuge & $B, L, S$ & 72 & 1.33 & 23.63 & - & - & - & - & - & - \\
\hline $\begin{array}{l}\text { Lyonia ovalifolia } \\
\text { (Wall.) Drude }\end{array}$ & Ericaceae & Wounds, boils & S & - & - & - & - & - & - & 153 & 3.55 & 54.97 \\
\hline $\begin{array}{l}\text { Ougeinia } \\
\text { oojeinensis Hochr. }\end{array}$ & Fabaceae & digestive troubles & G & 32 & 0.40 & 14.69 & 56 & 1.24 & 22.6 & - & - & - \\
\hline $\begin{array}{l}\text { Phyllanthus } \\
\text { embelica L. }\end{array}$ & Euphorbiaceae & Source of vitamin C & $\mathrm{F}$ & - & - & - & 44 & 0.81 & 19.02 & - & - & - \\
\hline $\begin{array}{l}\text { Prunus cerasoides } \\
\text { Buch.-Ham. ex D. } \\
\text { Don }\end{array}$ & Rosaceae & Swellings, contusions & B & - & - & - & - & - & - & 84 & 1.73 & 33.76 \\
\hline $\begin{array}{l}\text { Pyrus pashia } \\
\text { Buch.-Ham. ex D. } \\
\text { Don }\end{array}$ & Rosaceae & digestive disorders & $\mathrm{F}$ & - & - & - & - & - & - & 82 & 1.75 & 30.87 \\
\hline $\begin{array}{l}\text { Quercus } \\
\text { leucotrichophora } \\
\text { A. Camus }\end{array}$ & Fagaceae & $\begin{array}{l}\text { gonorrheal and digestive } \\
\text { disorders }\end{array}$ & G & - & - & - & - & - & - & 219 & 5.02 & 71.14 \\
\hline $\begin{array}{l}\text { Rhododendron } \\
\text { arboreum Sm. }\end{array}$ & Ericaceae & $\begin{array}{l}\text { digestive and respiratory } \\
\text { disorders }\end{array}$ & $F, B$ & - & - & - & - & - & - & 160 & 4.40 & 62.19 \\
\hline $\begin{array}{l}\text { Terminalia belerica } \\
\text { Roxb. }\end{array}$ & Combretaceae & Fruit is ingredient of Trifala & $\mathrm{F}$ & 32 & 1.28 & 20.34 & 32 & 0.74 & 11.43 & - & - & - \\
\hline $\begin{array}{l}\text { Terminalia } \\
\text { chebula Retz. }\end{array}$ & Combretaceae & Fruit is ingredient of Trifala & $\mathrm{F}$ & - & - & - & 32 & 1.34 & 14.19 & - & - & - \\
\hline $\begin{array}{l}\text { Terminalia } \\
\text { tomentosa (Roxb.) } \\
\text { Wight \&Arn. }\end{array}$ & Combretaceae & liver troubles & B & 24 & 0.57 & 15.09 & 36 & 1.34 & 17.34 & - & - & - \\
\hline \multicolumn{13}{|l|}{ Shrubs $\left(\right.$ ha $\left.^{-1}\right)$} \\
\hline $\begin{array}{l}\text { Adhatoda vasica } \\
\text { Nees in Wallich, } \\
\text { Pl. Asiat. Rar. }\end{array}$ & Acanthaceae & $\begin{array}{l}\text { cough, cold, pulmonary } \\
\text { affections, bronchitis and fever }\end{array}$ & $F, L, T$ & 364 & 0.041 & 60.79 & 394 & 0.062 & 36.45 & - & - & - \\
\hline $\begin{array}{l}\text { Berberis asiatica } \\
\text { Roxb. }\end{array}$ & Berberidaceae & ophthalmic & $\mathrm{R}$ & - & - & - & - & - & - & 275 & 0.034 & 77.80 \\
\hline $\begin{array}{l}\text { Calotropis procera } \\
\text { (Aiton). W.T. Aiton }\end{array}$ & Asclepiadaceae & expectorant, cough, cold, asthma & $R, F$ & 92 & 0.007 & 16.96 & - & - & - & - & - & - \\
\hline $\begin{array}{l}\text { Colebrookea } \\
\text { oppositifolia Sm. }\end{array}$ & Lamiaceae & wounds & L & 72 & 0.008 & 13.48 & - & - & - & - & - & - \\
\hline $\begin{array}{l}\text { Cotoneaster } \\
\text { bacillaris Wall. } \\
\text { Kurz ex Lindl. } \\
\end{array}$ & Rosaceae & scabies and rheumatic arthritis & L & - & - & - & - & - & - & 72 & 0.009 & 26.83 \\
\hline $\begin{array}{l}\text { Indigofera } \\
\text { gerardiana Wall. } \\
\text { ex Baker }\end{array}$ & Fabaceae & diarrhea, dysentery and cough. & L & & & & 252 & 0.063 & 29.25 & - & - & - \\
\hline $\begin{array}{l}\text { Leptodermis } \\
\text { lanceolata Wall. }\end{array}$ & Rubiaceae & migraines & $B$ & - & - & - & - & - & - & 116 & 0.011 & 28.79 \\
\hline $\begin{array}{l}\text { Prinsipia utilis } \\
\text { Royle }\end{array}$ & Rosaceae & rheumatic pains, diarrhea & $S, B$ & - & - & - & - & - & - & 180 & 0.042 & 41.86 \\
\hline
\end{tabular}


Table 2 Density, TBC (total basal cover), IVI (importance value index) of ethnomedicinal plants (Continued)

\begin{tabular}{|c|c|c|c|c|c|c|c|c|c|c|c|c|}
\hline $\begin{array}{l}\text { Rhus parviflora } \\
\text { Roxb. }\end{array}$ & Anacardiaceae & Cholera & $L$ & & & & 284 & 0.113 & 36.22 & 88 & 0.015 & 26.49 \\
\hline $\begin{array}{l}\text { Woodfordia } \\
\text { fructicosa } L \text {. }\end{array}$ & Lythraceae & febrifuge & $L$ & & & & 316 & 0.092 & 37.33 & - & - & - \\
\hline \multicolumn{13}{|l|}{ Herbs $\left(\mathrm{m}^{2}\right)$} \\
\hline $\begin{array}{l}\text { Achyranthes } \\
\text { aspera L }\end{array}$ & Amaranthaceae & $\begin{array}{l}\text { malarial fever, delivery, dropsy, } \\
\text { bronchitis }\end{array}$ & WP & 0.36 & 0.025 & 15.33 & 0.08 & 0.009 & 9.08 & - & - & - \\
\hline $\begin{array}{l}\text { Aerva } \\
\text { sanguinolenta (L.) } \\
\text { Blume }\end{array}$ & Amaranthaceae & Diuretic, demulcent. & WP & 0.37 & 0.06 & 25.68 & 0.12 & 0.011 & 9.08 & - & - & - \\
\hline $\begin{array}{l}\text { Ageratum } \\
\text { conyzoides L. }\end{array}$ & Asteraceae & sores, cuts, skin ailments & WP & - & - & - & 0.15 & 0.016 & 10.11 & - & - & - \\
\hline $\begin{array}{l}\text { Ajuga } \\
\text { brachystemon } \\
\text { Maxim. }\end{array}$ & Lamiaceae & febrifuge & $\mathrm{L}$ & 0.17 & 0.009 & 9.88 & - & - & - & - & - & - \\
\hline $\begin{array}{l}\text { Anagallis arvensis } \\
\mathrm{L} \text {. }\end{array}$ & Primulaceae & $\begin{array}{l}\text { leprosy, dropsy,cerebral } \\
\text { affections }\end{array}$ & WP & - & - & - & - & - & - & 0.26 & 0.004 & 9.19 \\
\hline Barleria prionitis L. & Acanthaceae & Cough, cold & $\mathrm{R}, \mathrm{B}$ & 0.21 & 0.004 & 8.86 & 0.20 & 0.006 & 11.44 & - & - & - \\
\hline $\begin{array}{l}\text { Bidens bipinnata } \\
\text { L. }\end{array}$ & Asteraceae & Leprosy, cures & $L$ & 0.17 & 0.023 & 11.91 & - & - & - & - & - & - \\
\hline $\begin{array}{l}\text { Boerhavia diffusa } \\
\text { L. }\end{array}$ & Nyctaginaceae & Asthma, bronchitis, energy tonic & WP & 0.32 & 0.007 & 11.86 & 0.26 & 0.007 & 13.35 & - & - & - \\
\hline $\begin{array}{l}\text { Commelina } \\
\text { benghalensis L. }\end{array}$ & Commelinaceae & Dysentery, swelling, ache. & WP & 0.21 & 0.026 & 13.12 & 0.19 & 0.001 & 13.51 & - & - & - \\
\hline $\begin{array}{l}\text { Cynodon dactylon } \\
\text { (L.) Pers. }\end{array}$ & Poaceae & fever & $\mathrm{R}$ & 0.42 & 0.002 & 11.49 & 0.25 & 0.032 & 21.42 & - & - & - \\
\hline $\begin{array}{l}\text { Cynoglossum } \\
\text { glochidiatum Wall. } \\
\text { ex Benth. }\end{array}$ & Boraginaceae & Dyspepsia, digestive. & $\mathrm{R}$ & 0.19 & 0.024 & 13.39 & 0.28 & 0.032 & 21.87 & - & - & - \\
\hline $\begin{array}{l}\text { Desmodium } \\
\text { elegans DC. }\end{array}$ & Fabaceae & carminatives & $\mathrm{R}$ & 0.20 & 0.23 & 11.38 & - & - & - & - & - & - \\
\hline Euphorbia hirta L. & Euphorbiaceae & $\begin{array}{l}\text { bronchial infection, asthma, } \\
\text { warts }\end{array}$ & WP & - & - & - & 0.22 & 0.006 & 11.96 & - & - & - \\
\hline $\begin{array}{l}\text { Geranium } \\
\text { ocellatum } \\
\text { Cambess. }\end{array}$ & Geraniaceae & liver troubles, fever & WP & - & - & - & - & - & - & 0.35 & 0.033 & 18.76 \\
\hline $\begin{array}{l}\text { Imperata } \\
\text { cylindrica L. }\end{array}$ & Poaceae & tonic & $\mathrm{R}$ & - & - & - & - & - & - & 0.33 & 0.004 & 9.18 \\
\hline $\begin{array}{l}\text { Launaea } \\
\text { asplenifolia Hook. } \\
\text { f. }\end{array}$ & Asteraceae & diarrhea & $\mathrm{R}$ & - & - & - & - & - & - & 0.35 & 0.003 & 11.94 \\
\hline $\begin{array}{l}\text { Leucus indica (L.) } \\
\text { R. Br. Ex Vatke }\end{array}$ & Lamiaceae & Wound, sores & $L$ & 0.16 & 0.026 & 11.05 & - & - & - & - & - & - \\
\hline Mentha arvensis L. & Lamiaceae & Vomiting, indigestion & WP & - & - & - & - & - & - & 0.25 & 0.031 & 14.92 \\
\hline $\begin{array}{l}\text { Micromeria biflora } \\
\text { (Buch.-Ham. ex D. } \\
\text { Don) Benth. }\end{array}$ & Lamiaceae & gastroenteritis & $L$ & - & - & - & 0.45 & 0.015 & 17.32 & - & - & - \\
\hline Origanum vulgare & Lamiaceae & bronchitis, colic, diarrhea & WP & 0.27 & 0.07 & 24.74 & - & - & - & - & - & - \\
\hline $\begin{array}{l}\text { Oxalis corniculata } \\
\text { (DC.) Raeusch }\end{array}$ & Oxalidaceae & Cataract, conjunctivitis & L & 0.33 & 0.002 & 11.49 & 0.025 & 0.032 & 21.42 & - & - & - \\
\hline $\begin{array}{l}\text { Phyllanthus } \\
\text { amarus } \\
\text { Schumach. \& } \\
\text { Thonn. }\end{array}$ & Euphorbiaceae & $\begin{array}{l}\text { astringent, stomachic, diuretic, } \\
\text { febrifuge }\end{array}$ & WP & 0.42 & 0.002 & 14.07 & 0.22 & 0.003 & 10.24 & - & - & - \\
\hline $\begin{array}{l}\text { Pimpinella } \\
\text { diversifolia DC. }\end{array}$ & Apiaceae & Cough, cold, digestive disorders & WP & - & - & - & 0.37 & 0.004 & 15.13 & - & - & - \\
\hline
\end{tabular}


Table 2 Density, TBC (total basal cover), IVI (importance value index) of ethnomedicinal plants (Continued)

\begin{tabular}{|c|c|c|c|c|c|c|c|c|c|c|c|c|}
\hline $\begin{array}{l}\text { Ranunculus } \\
\text { sceleratus } \mathrm{L} \text {. }\end{array}$ & Ranunculaceae & Vermifuge, skin disorders & WP & 0.18 & 0.004 & 10.53 & - & - & - & - & - & - \\
\hline $\begin{array}{l}\text { Roylae cinerea (D. } \\
\text { Don) Baill. }\end{array}$ & Lamiaceae & malarial fever & L & 0.17 & 0.014 & 9.13 & - & - & - & - & - & - \\
\hline $\begin{array}{l}\text { Rumex hastatus } \\
\text { D. Don }\end{array}$ & Polygonaceae & Cuts, wounds,check bleeding & $\mathrm{L}$ & 0.20 & 0.03 & 13.80 & 0.10 & 0.005 & 11.49 & - & - & - \\
\hline $\begin{array}{l}\text { Saponaria } \\
\text { vaccaria } \mathrm{L} \text {. }\end{array}$ & Caryophyllaceae & bile complaints & WP & - & - & - & - & - & - & 0.27 & 0.005 & 12.80 \\
\hline Sida acuta Burm.f & Malvaceae & Demulcent, diuretic, leucorrhoea & $L, R$ & 0.34 & 0.054 & 21.06 & - & - & - & - & - & - \\
\hline$\overline{\text { Sida cordifolia } \mathrm{L} \text {. }}$ & Malvaceae & dyspepsia, astringent, diuretic & $S, R$ & 0.44 & 0.101 & 32.31 & 0.155 & 0.004 & 39.54 & - & - & - \\
\hline $\begin{array}{l}\text { Swertia } \\
\text { angustifolia Buch.- } \\
\text { Ham. ex D.Don }\end{array}$ & Gentianaceae & febrifuge & WP & - & - & - & - & - & - & 0.23 & 0.023 & 14.92 \\
\hline $\begin{array}{l}\text { Tridax } \\
\text { procumbens } \mathrm{L} \text {. }\end{array}$ & Asteraceae & Wounds, cuts & WP & 0.39 & 0.002 & 13.37 & 0.34 & 0.043 & 27.35 & - & - & - \\
\hline $\begin{array}{l}\text { Vernonia } \\
\text { anthelmintica (L) } \\
\text { Willd. }\end{array}$ & Asteraceae & $\begin{array}{l}\text { intestinal disorders, fever, skin } \\
\text { ailments }\end{array}$ & WP & - & - & - & - & - & - & 0.26 & 0.053 & 21.84 \\
\hline $\begin{array}{l}\text { Potentilla } \\
\text { gerardiana Lindey } \\
\text { ex Lehmann }\end{array}$ & Rosaceae & wounds & $\mathrm{R}$ & - & - & - & - & - & - & 0.23 & 0.05 & 14.9 \\
\hline
\end{tabular}

$\mathrm{W}=$ wood; $\mathrm{B}=$ Bark; F = Fruit; $\mathrm{L}=$ Leaf; $\mathrm{S}=$ Seed; $\mathrm{G}=$ Gum; F = Flower; $\mathrm{T}=$ Twigs; $\mathrm{R}=$ Root; WP = Whole Plant

The consensus factor was calculated as follows

$$
\text { Fic }=\mathrm{N}_{\mathrm{ur}}-\mathrm{N}_{\mathrm{t}} /\left(\mathrm{N}_{\mathrm{ur}}-1\right)
$$

The resulting factor ranges between 0 to 1 , where a high value indicates for a high rate of informant consensus. $\mathrm{N}_{\mathrm{ur}}$ is the number of use-reports of informant's for a particular illness, where a use-report is a single record of the use of a plant mentioned by an individual, and $\mathrm{N}_{\mathrm{t}}$ refers to the number of taxa (species) used for a particular illness category by all informants.

\section{Results and Discussion}

\section{Ethnomedicinal uses}

Ethnobotany is not new to India [26] with over 400 different tribal and other ethnic groups $[27,28]$. Ethnobotanical information on medicinal plants and their uses by indigenous cultures is useful not only for the conservation of traditional knowledge and biodiversity, but also to promote community health care, and might serve in drug development. The information can provide a guide for drug development, assuming that a plant that has been used by indigenous people over a long period of time may well have an allopathic application $[29,30]$.

Table 2 provides the scientific names for all plants collected, as well as information on the parts used. Overall 14 trees, 10 shrubs, and 33 herbs were identified. These plants were used to treat a total of 47 diseases, ranging from simple to highly complicated, including asthma and respiratory problems. The greatest number of plants (7) was used for digestive disorders, followed by fever (6) and bronchitis (5). A single species was recorded to treat each of the following ailments: warts, vomiting, carminative, pains, boils, and much other species (Figure 2).

A comparative study in Bhotiya tribal communities in the Central Himalaya found that eighty-six plant species were identified as being used for treatment of 37 common ailments [31].

A study on the status of medicinal plants in Uttarakhand Himalaya [32] found a total of 243 medical herbal formulations prepared by Vaidyas (healers) treating 73 different ailments. Plants were used as the major ingredients for these medical formulations. A total of 156 medicinal plant species were documented during the survey. Of these $55 \%$ were cultivated and $45 \%$ were wild collected.

The plants found in the present study are distributed among 30 plant families. The largest number of species (7) belonged to the Lamiaceae followed by Asteraceae, Rosaceae, and Fabaceae with five species each (Table 3). A study of medicinal plants in the trans-Himalayan arid zone of Mustang district, Nepal, also found the largest numbers of medicinal plants belonged to the Lamiaceae [33].

A field survey conducted in four different places of Kathmandu valley recorded thirty six medicinal plants used to treat ailments such as diarrhoea, stomach ache, gastritis, jaundice, bodyache, bleeding, etc. [34]. The results indicate that inhabitants of the Kathmandu valley still rely on traditional medicines for their primary health care. The indigenous knowledge of local traditional healers in the Kancheepuram district of 


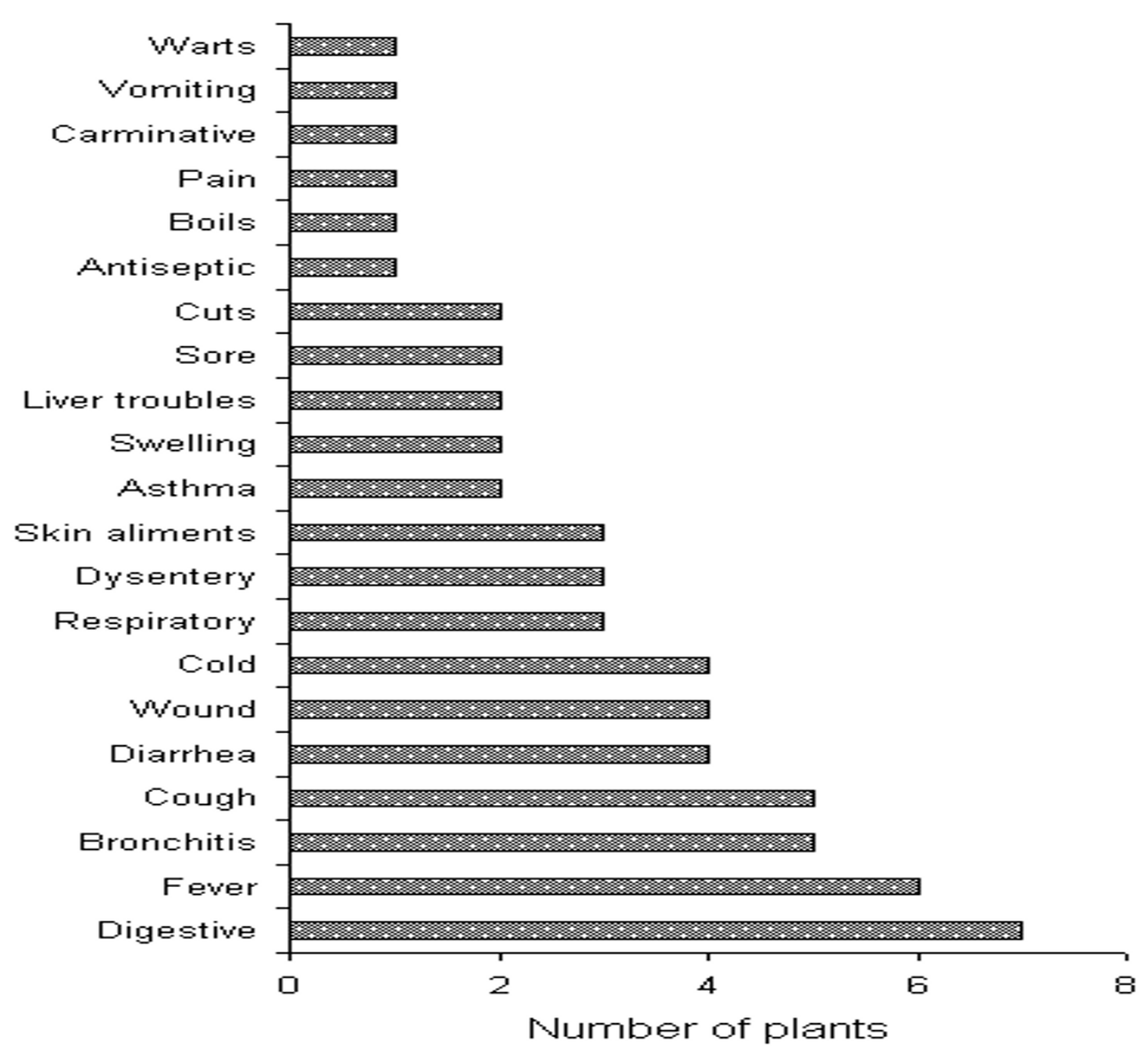

Figure 2 Number of plants used for different disease curing

Tamilnadu also showed that many people still continue to depend on medicinal plants at least for the treatment of common health problems [35].

In the present study most preparations used the whole plant, followed by leaf and roots (Figure 3). A study conducted in Chakrata Forest Division, Uttarakhand, showed a higher incidence of root, leaf, and bark use to treat various diseases [36].

Traditional medicines are a central component in health care systems in developing countries, where up to $80 \%$ of the population depends on traditional medical systems. The use of herbal medicines, is also increasing in developed countries, based on the belief that herbal remedies are safe because of their natural origin [37]. Globally, there are about 120 plant-derived drugs in professional use; three quarters of which are obtained from traditional medicinal plants [38]. Unfortunately, according to a recent report, almost one third of medicinal plant species could become extinct, with significant losses reported in China, India, Kenya, Nepal, Tanzania, and Uganda [39]. Greater losses are expected to occur in arid and semi-arid areas due to the impact of climate change, erosion, expansion of agricultural land, wood consumption, and exploitation of natural vegetation, increased global trade in natural resources, domestication, selection and grazing [40].

The traditional plant knowledge however is disappearing in many communities because of rapid socioeconomic and cultural change. The sustained use of this knowledge and its documentation is therefore essential.

\section{Ecological status}

In the present study, the distribution of species ranged from altitude $350 \mathrm{~m}$ to $2300 \mathrm{~m}$ m.a.s.l. The vegetation composition varied with changes in altitude. Plant resources distributed across complex landscapes 
Table 3 Distribution of herbs, shrubs and trees in different families

\begin{tabular}{|c|c|c|c|c|}
\hline Family & Herb & Shrub & Tree & Total \\
\hline Acanthaceae & 1 & 1 & - & 2 \\
\hline Amaranthaceae & 2 & - & - & 2 \\
\hline Apiaceae & 1 & - & - & 1 \\
\hline Asteraceae & 5 & - & - & 5 \\
\hline Boraginaceae & 1 & - & - & 1 \\
\hline Caryophyllaceae & 1 & - & - & 1 \\
\hline Commelinaceae & 1 & - & - & 1 \\
\hline Euphorbiaceae & 2 & - & - & 2 \\
\hline Rosaceae & 1 & 2 & 2 & 5 \\
\hline Fabaceae & 1 & 1 & 3 & 5 \\
\hline Gentianaceae & 2 & - & - & 2 \\
\hline Lamiaceae & 6 & 1 & - & 7 \\
\hline Malvaceae & 2 & - & - & 2 \\
\hline Nyctaginaceae & 1 & - & - & 1 \\
\hline Oxalidaceae & 1 & - & - & 1 \\
\hline Poaceae & 2 & - & - & 2 \\
\hline Polygonaceae & 1 & - & - & 1 \\
\hline Ranunculaceae & 1 & - & - & 1 \\
\hline Primulaceae & 1 & - & - & 1 \\
\hline Rutaceae & - & - & 1 & 1 \\
\hline Apocynaceae & - & - & 1 & 1 \\
\hline Ericaceae & - & - & 2 & 2 \\
\hline Euphorbiaceae & - & - & 1 & 1 \\
\hline Fagaceae & - & - & 1 & 1 \\
\hline Combretaceae & - & - & 3 & 3 \\
\hline Berberidaceae & - & 1 & - & 1 \\
\hline Asclepiadaceae & - & 1 & - & 1 \\
\hline Rubiaceae & - & 1 & - & 1 \\
\hline Anacardiaceae & - & 1 & - & 1 \\
\hline Lythraceae & - & 1 & - & 1 \\
\hline Total & 33 & 10 & 14 & 57 \\
\hline
\end{tabular}

modified for human needs [11]. For example, conservation agencies recognize geographically uneven occurrences of high species richness and rare and endemic plants in their designation of biodiversity hotspots [40-42] or ecoregions $[43,44]$. These localities are then prioritized by the degree to which human activities threaten existing patterns [45]. Diversity patterns at different geographic scales, however, may be created or degraded by physical-environmental conditions and human-historical processes that influence resource availability and habitat heterogeneity [46,47].

In this study, we focused on the ecology and ethnomedicine of woody and herbaceous plants. These plants are distributed in highly-fragmented habitats, and are potentially threatened. Among the high peaks of the Himalaya, local inhabitants were found to inflict a great deal of pressure on medicinal plant populations because at higher altitudes health care facilities were almost nonexistent, and people met their medicinal requirements with forest products. At lower altitudes, people also used medicinal plants, but owing to better infrastructure, they also used nearby health centers for the treatment various diseases.

To promote a conservation agenda, it is important to understand how local communities use and manage natural resources. Studies in ethnobiology (including ethnobotany) and traditional ecological knowledge are known to serve as significant bridges between conservation scientists and local communities. These studies help to explain how local communities relate to their environment and hence, suggest ways to promote their active involvement in natural resource conservation [48].

The ecological information of plants is given in Table 2. A total of 57 species were recorded from all three regions (tropical, sub-tropical and temperate). Among the trees, Acacia catechu, Aegal marmelose, Ougeinia oojeinensis, Terminalia belerica, and Terminalia tomentosa were common in the tropical and sub-tropical regions. Acacia catechu was dominant in the tropical and sub-tropical regions. Ougeinia oojeinensis was the least common tree in the tropical region and Terminalia belerica in sub-tropical region. In the temperate region, Quercus leucotrichophora was dominant and Pyrus pashia least dominant. Other associated species are shown in Table 2 . In the shrub layer, a total of 10 species were recorded from all regions: Three species were found in tropical areas, 4 in sub-tropical areas, and 5 in temperate areas. Adhatoda vasica was found in both tropical and sub-tropical regions, while Rhus parviflora occurred in both sub-tropical and temperate regions. Among the 33 species of herbs, Sida cordifolia was dominant in tropical and sub-tropical region while least dominant herb in the tropical region was Barleria prionitis, and in the sub-tropical region Achyranthes aspera and Aerva sanguinolenta. In the temperate areas, the dominant and least dominant species were Vernonia anthelmintica and Imperata cylindrical respectively. Other associated herbs are given in Table 2.

The density and total basal cover of trees, shrubs and herbs in the tropical, sub-tropical and temperate regions is shown in Figures $4 \mathrm{a}, 4 \mathrm{~b}$, and $4 \mathrm{c}$. In the tree layer the highest value of density (698 trees $\mathrm{ha}^{-1}$ ) and total basal cover $\left(16.45 \mathrm{~m}^{2} \mathrm{ha}^{-1}\right)$ was in temperate region followed by sub-tropical and tropical regions (Figure 4a). In the shrub layer the density and total basal cover was highest in sub-tropical region and lowest in the tropical region (Figure $4 \mathrm{~b}$ ). In the herb layer the trend of density and total basal cover was inverse to the tree layer and highest in the tropical region, followed by the sub-tropical and temperate regions (Figure 4c). The trend of tree density and total basal cover increased with increasing altitudes. Shrubs were increasing in the sub-tropical 


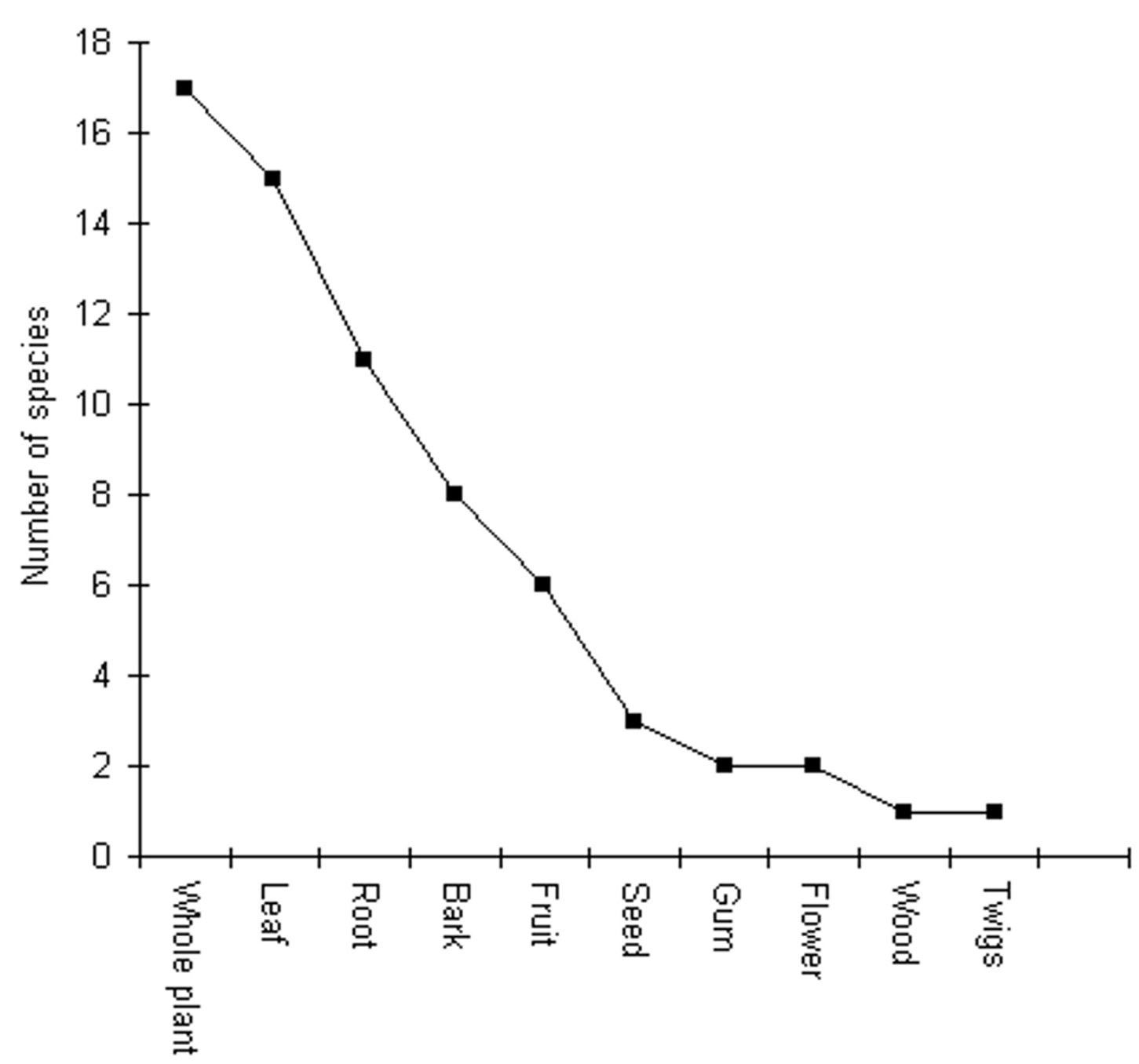

Figure 3 Number of plants parts used for disease curing

region. The dependency of the villagers on medicinal plants increased with increasing altitudes due to increasing lack in healthcare facilities. Herb density and total basal cover were reduced with altitude, which could be the main effect of exploitation of these medicinal herbs for human health.

Most of the informants suggested that medicinal plants are an important source for daily healthcare and the associated knowledge was traditionally transmitted. They also suggested that these species help maintain the ecological balance of the area by decreasing soil erosion and increasing moisture in the soil, thus improving conditions for human and livestock needs. Most of the respondent said however that they did not apply any management or paid any attention to conservation needs of the species because of lack ecological knowledge. People were well aware that deforestation, overgrazing, and overexploitation of the species in a particular region may lead to the extinction of this valuable resource. The changing ecological situation was recognized as a main reason for severe problems like forest fires, erosion and drought, as well as for the disappearance of important medicinal species. The expansion of agriculture, and logging was mentioned as clearly reducing the population of highly valuable medicinal plants.

\section{Consensus survey of medicinal plants}

The consensus survey indicated that six plant species were used most commonly for individual diseases, and therefore the informant's consensus index factor was high (1.0). Two taxa were often used for five other diseases (the index factor range was 0.75 to 0.98 ). Digestive diseases were cured with the highest number of taxa (7) and its consensus index factor was 0.84 (Table 4). The local population had a very high level 


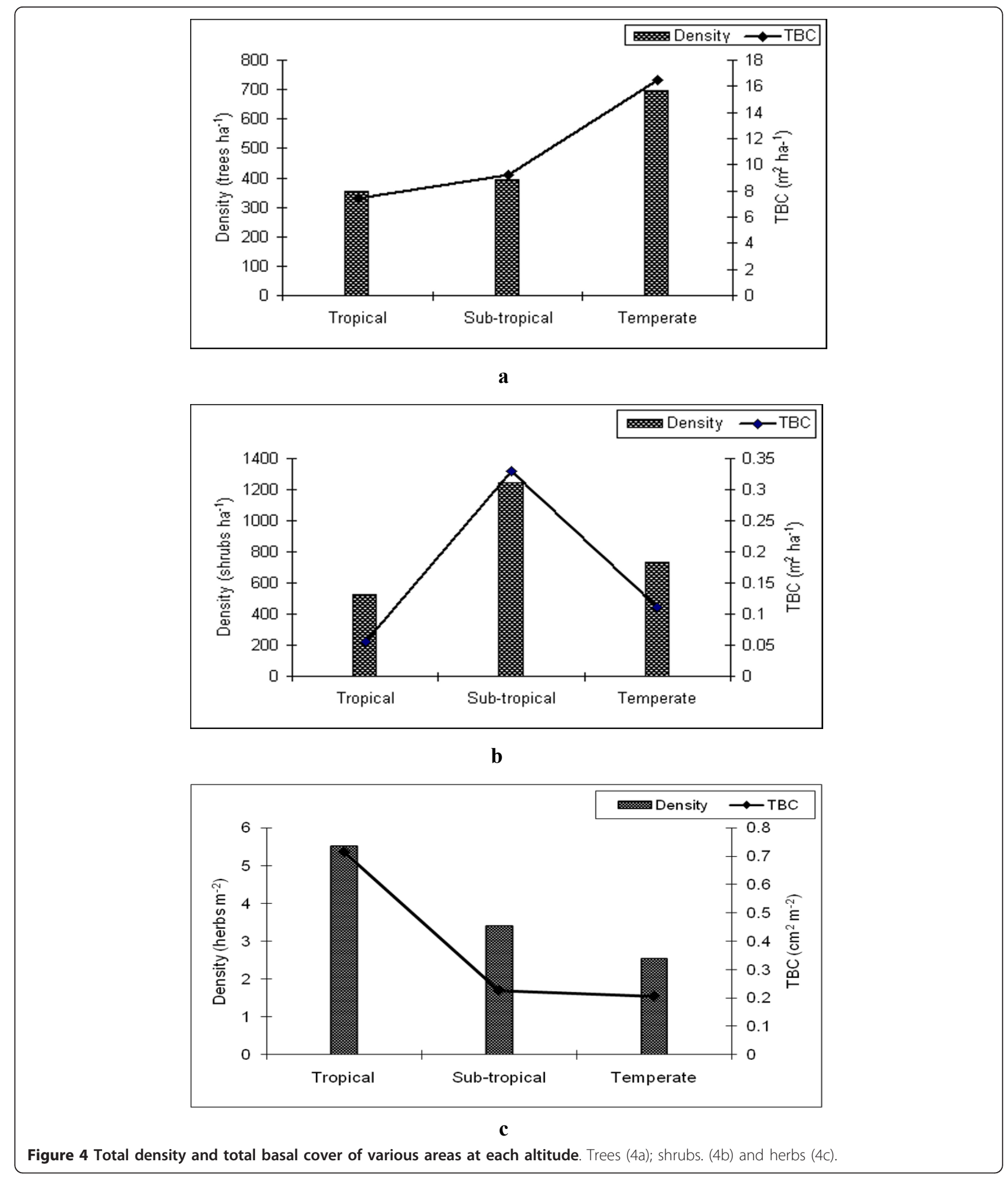

of agreement on the usages of plants for specific ailments. The index value was high for warts, vomiting, carminative, pain, boils and antiseptic uses (1.0), and lowest for bronchitis (0.33) were five (5). Ragupathy et al. [25] published first consensus analysis research for aboriginal group in India. The consensus report of northeast India is also carried out by Sajem and Gosai [49]. 
Table 4 Consensus index for ethnomedicinal plants

\begin{tabular}{|c|c|c|c|}
\hline $\begin{array}{l}\text { Illness } \\
\text { category }\end{array}$ & $\begin{array}{l}\text { Number } \\
\text { taxa used } \\
\left(\mathrm{N}_{\mathrm{t}}\right)\end{array}$ & $\begin{array}{l}\text { Number of use- } \\
\text { reports }\left(\mathrm{N}_{\mathrm{ur}}\right)\end{array}$ & $\begin{array}{l}\text { Informant's consensus } \\
\text { index factor }\left(F_{i c}\right)^{a}\end{array}$ \\
\hline Warts & 1 & 22 & 1.0 \\
\hline Vomiting & 1 & 29 & 1.0 \\
\hline Carminative & 1 & 15 & 1.0 \\
\hline Pain & 1 & 40 & 1.0 \\
\hline Boils & 1 & 21 & 1.0 \\
\hline Antiseptic & 1 & 39 & 1.0 \\
\hline Cuts & 2 & 51 & 0.98 \\
\hline Sores & 2 & 39 & 0.92 \\
\hline $\begin{array}{l}\text { Liver } \\
\text { troubles }\end{array}$ & 2 & 5 & 0.75 \\
\hline Swelling & 2 & 61 & 0.98 \\
\hline Asthma & 2 & 15 & 0.93 \\
\hline $\begin{array}{l}\text { Skin } \\
\text { aliments }\end{array}$ & 3 & 25 & 0.92 \\
\hline Dysentery & 3 & 31 & 0.93 \\
\hline Respiratory & 3 & 10 & 0.78 \\
\hline Cold & 4 & 13 & 0.75 \\
\hline Wound & 4 & 42 & 0.93 \\
\hline Diarrhea & 4 & 16 & 0.80 \\
\hline Cough & 5 & 13 & 0.67 \\
\hline Bronchitis & 5 & 7 & 0.33 \\
\hline Fever & 6 & 16 & 0.67 \\
\hline Digestive & 7 & 39 & 0.84 \\
\hline
\end{tabular}

${ }^{a} F_{i c}=N_{u r}-N_{t} /\left(N_{u r}-1\right)$, providing a value between 0 and 1 , where high value indicates a high rate of informant consensus.

\section{Conclusions}

The results of this study indicate that medicinal plants are used frequently by local people in the region. Some of the plants are already under threat because of overexploitation, including clearing land for agriculture, encroachment and abrupt change in environmental conditions. The majority bulk of the inhabitants seem to be unaware of the great threat to medicinal plants growing in the wild. The data presented here helps to fill this educational and awareness gap. In particular, the importance of these medicinal plants for treating various diseases must be emphasized, and their preservation and sustainable use must be included in future conservation strategies.

\section{Acknowledgements}

The authors are thankful to the communities participating in the study and sharing their knowledge.

\section{Author details}

'Department of Forestry, HNB Garhwal University, Srinagar Garhwal, Uttarakhand, India. ${ }^{2}$ William L. Brown Center, Missouri Botanical Garden, St. Louis, MO 63110, USA.

\section{Authors' contributions}

MK designed the study, preformed the field survey, and prepared the draft manuscript, MAS analyzed the data and prepared the draft manuscript, RWB revised the manuscript and data analysis. All authors read and approved the final manuscript.

\section{Competing interests}

The authors declare that they have no competing interests.

Received: 6 December 2010 Accepted: 19 October 2011

Published: 19 October 2011

\section{References}

1. Dhar U, Rawal RS, Upreti J: Setting priorities for conservation of medicinal plants - A case study in the Indian Himalaya 2000, 57-65.

2. Singh DK, Hajara PK: Floristic diversity. In Biodiversity Status in the Himalaya New Delhi: Britis Council; 1996, 23-38.

3. Samant SS, Dhar U, Palni LMS: Medicinal Plants of Indian Himalaya: Diversity Distribution Potential Value. Almora: G.B Pant Institute of Himalayan Environment and Development; 1998.

4. Kala CP: Revitalizing traditional herbal therapy by exploring medicinal plants: A casestudy of Uttaranchal State in India. In Indigenous Knowledge: Transforming the Academy, Proceedings of an International Conferences Pennsylvania: Pennsylvania State University; 2004, 15-21.

5. Verma AK, Kumar M, Bussmann RW: Medicinal plants in an urban environment: the medicinal flora of Banares Hindu University, Varanasi, Uttar Pradesh. Journal of Ethnobiology and Ethnomedicine 2007, 3:35.

6. Harshburger JW: Purpose of Ethnobotany. Botanical Gazette 1896, 21:146-154.

7. Azaizeh H, Fulder S, Khalil K, Said O: Ethonomedicinal knowledge of local Arab Practitioners in the Middle East Region. Fitoterapia 2003, 74:98-108

8. Anonmous: Amruth, August, FRLHT, Banglore -10. 1997.

9. Berkes F, Colding J, Folke C: Rediscover of traditional ecological knowledge as adaptive management. Ecological application 2000, 10:1251-1262.

10. Huntingto HP: Using traditional ecological knowledge in science: methods and application. Ecological Applications 2000, 10:1270-1274.

11. Cunningham AB: Applied Ethnobotany. People, Wild Plant Use and Conservation. Earthscan, London; 2001, 300.

12. Gunderson LH, Holling CS: Panarchy: undrestandig trasnformatons in human and natural systems. Island Press, Washington, D.C., USA; 2002.

13. Hussain F, Sher H, Ibrar Mohammad, Durrani MJ: Ethnobotanical uses of plants of District Swat, Pakistan. Pak J PI Sci 2005, 11(2):137-158.

14. Singh VK, Ali ZA, Siddiqui MK: Folk medicinal plants of Garhwal and Kumaon forest of Uttar Pradesh, India. Hamdard Medicus 1997, 40:35-47.

15. Gupta MP, Corea MD, Soils PN, Jones A, Galdames C: Medicinal Plants inventory of Kuna Indians: Part1. Journal Ethnopharmacology 1995, 40:77-109

16. Siwakoti M, Siwakoti S: Ethnomedicianl uses of plants among limbu of Morang district, Nepal. Ecoprint 1998, 5:79-84.

17. Durrani MJ, Malik AM, Hussain F: Folk medicinal plants of Nushki, District Chaghi, Pakistan. Jour Sci \& Technol 2003, 27(1\&2):45-52

18. Sher H, Midrarullah AU, Khan HF, Ahmad S: Medicinal plants of Udigram, District Swat, Pakistan. Pak J Forest 2003, 53(1):65-74.

19. Dash PK, Santilata Sahoo, Subhasisa Bal: Ethnobotanical Studies on Orchids of Niyamgiri Hill Ranges, Orissa, India Ethnobotanical Leaflets. 2008, 12:70-78

20. Tantray MA, Tariq KA, Mir MM, Bhat MA, Shawl AS: Ethnomedicinal survey of shopian, Kashmir (J\&K), India Ethnomedicinal survey of shopian, Kashmir (J\&K), India. Asian Journal of Traditional Medicines 2009, 4(1):1-6.

21. Curtis JT, Mc Intosh RP: The Interrelation of certain analytic and synthetic phytosociological characters. Ecology 1950, 31:434-455.

22. Curtis JT: The Vegetation of Wisconsin An ordination of plant communities, University Wisconsin Press, Madison Wisconsin; 1959, 657

23. Gaur RD: Flora of the District Garhwal North West Himalaya with Ethnobotnaical notes Tran media Publication, Srinagar Garhwal; 1999.

24. Trotter R, Logan M: Informant consensus: a new approach for identifying potentially effective medicinal plants. In Plants inindigenous medicine and diet: biobehavioural approaches. Edited by: Etkin NL. Bedfort hills, New York: Redgrave Publishers; 1986:91-112.

25. Ragupathy S, Steven NG, Maruthakkutti M, Velusamy B, Huda MMUI: Consensus of the 'Malasars' traditional aboriginal knowledge of medicinal plants in the Velliangiri holy hills, India. Journal of Ethnobiology and Ethnomedicine 2008, 4:8. 
26. Khoshoo TN: Plant diversity in Himalaya. Conservation and utilization. G. B. Pant memorial Lecture II G.B. Pant Institute of Himalayan Environment and Development, Koshi-Katarmal, Almora, India; 1992.

27. Jain SK: Dictionary of Indian Folk Medicine and Ethnobotany. Deep publications, Paschim Vihar, New Delhi; 1991.

28. Mukesh Joshi, Munesh Kumar, Bussmann RW: Ethnomedicinal Uses of Plant Resources of the Haigad Watershed in Kumaun Himalaya. Journal of Medicinal and Aromatic Plant Science and Biotechnology 2010, 4(1):43-46.

29. Farnsworth NR: Ethnopharmacology and future drug development: The North American experience. J Ethnopharmacol 1993, 38:145-152.

30. Khan Ajaib M, Zaheer-ud-din Khan N, Wahab M: Ethnobotanical studies on useful shrubs of district Kotli, Zzad Jammu \& Kashmir, Pakistan. Pak J Bot 2010, 42(3):1407-1415.

31. Phondani PC, Maikhuri RK, Rawat LS, Farooquee NA, Kala CP, Vishvakaram SCR, Rao KS, Saxena KG: Ethnobotanical useds of plants among the Bhotiya tribal communities of Niti valley in central Himalayan, India. Ethnobotany Research \& Applications 2010, 8:233-244.

32. Kala CP: Current status of Medicinal plants used by traditional Vaidys in Uttaranchal Sate of India. Ethnobotany Research and Application 2005, 267-278.

33. Shandesh Bhattarai, Chaudhary RP, Quave CL, Taylor RSL: The use of medicinal plants in the transhimalayan arid zone of Mustang district, Nepal. Journal of Ethnobiology and Ethnomedicine 2010, 6:14.

34. Acharya KP, Rokaya MB: Ethnobotanical survey of medicinal plants traded in the streets of Kathmandu Valley. Scientific World 2005, 3(3):44-48.

35. Muthu C, Ayyanar M, Raja N, Ignacimuthu S: Medicinal plants used by traditional healers in Kancheepuram District of Tamil Nadu, India. Journal of Ethnobiology and Ethnomedicine 2006, 2:43.

36. Dobhal P, Smriti Sawan, Sharma N: Studies on medicinal plants of tow villages of Chakrata Forest Division (Uttarakhand). Annals of Forestry 2007, 15(2):351-357.

37. Jacobsson I, Jönsson AK, Gredén B, Hägg S: Spontaneously reported adverse reactions in association with complementary and alternative medicine substances in Sweden. Pharmacoepidemiology and drug safety 2009, 18:1039-1047.

38. Marles RJ: Prairie medicinal and aromatic plants. Conference- Olds, Alberta; 1996.

39. Hamilton A: Medicinal plant extinction 'a quiet disaster'. New Scientist 2009.

40. Ayad MA: Case studies in the conservation of biodiversity degradation sand threats. Journal of Arid Environments 2003, 54:165-182.

41. Myers N, Mittermeier RA, Mittermeier CG, de Fonesca AB, Kent J: Biodiversity hotspots for conservation priorities. Nature 2000, 403:853-858.

42. Myers N: Biodiversity hotspots revisited. Bio Science 2003, 53:916-917.

43. Olson DM, Dinerstein E: The Global 200: A representations approach to conserving the Earth's most biologically valuable ecoregions. Conservation Biology 1998, 12(3):502-515.

44. World Wildlife Fund: Eastern Arc mountains- a global ecoregion 2007 [http:// wwf.panda.org/about_our_earth/ecoregions/easternarc_montane_forests. cfm], (verified on June 26, 2009).

45. Brooks TM, Mittermeier RA, Mittermeier CG, da Fonseca GAB, Rylands $A B$, Konstant WR, Flick P, Pilgrim J, Oldfield S, Magin G, Hilton-Taylor C: Habitat loss and extinction in the hotspots of biodiversity. Conservation Biology 2002, 16:909-923.

46. Huston MA: Biological Diversity: The coexistence of species on changing landscapes Cambridge University Press, Cambridge; 1994.

47. Posey DA: Indigenous knowledge in the conservation and use of world forests. Pp. 59-77 in. In World Forests for the Future: Their use and conservation. Edited by: Ramakrishna K, Woodwell GM. Yale University Press, New Haven, $C$; 1993:.

48. Duchelle AE: Observations on natural resource use and conservation by the Shuar in Ecuador's Cordillera del Condor. Ethnobotany Research \& Applications 2007, 5:005-023.

49. Sajem AL, Gosai K: Traditional use of medicinal plants by the Jaintia tribes in North Cachar hills district of Assam, northeast India. Journal of Ethnobiology and Ethnomedicine 2006, 2:33.

doi:10.1186/1746-4269-7-32

Cite this article as: Kumar et al:: Ethnomedicinal and ecological status of plants in Garhwal Himalaya, India. Journal of Ethnobiology and

Ethnomedicine 2011 7:32.

\section{Submit your next manuscript to BioMed Central and take full advantage of:}

- Convenient online submission

- Thorough peer review

- No space constraints or color figure charges

- Immediate publication on acceptance

- Inclusion in PubMed, CAS, Scopus and Google Scholar

- Research which is freely available for redistribution 